\title{
Pertussis: A Global Perspective
}

\author{
Halperin $\mathrm{SA}^{1 *}$
}

1. Canadian Center for Vaccinology, Departments of Pediatrics and Microbiology \& Immunology, Dalhousie University, IWK Health Centre, Halifax, NS

Corresponding author: scott.halperin@dal.ca

Over the past several years, outbreaks of pertussis have been reported from a number of jurisdictions including the United States ${ }^{1-4}$, Australia ${ }^{5}$, the United Kingdom ${ }^{6}$ and Canada. In the United States, over 9000 cases were reported in California in $2010^{7}$. In 2012, large outbreaks were reported in the states of Washington, Minnesota, and Wisconsin. Indeed, 49 of 50 states reported increases in pertussis in 2012 compared to 2011; only California which had its outbreak in 2010, reported a decrease in incidence ${ }^{8}$. In Australia, the overall incidence of pertussis in 2009 rose to $>140 / 100,000$, exceeding rates in most other industrialized countries ${ }^{9}$. In the UK, increased numbers of infant deaths were observed in association with an increase in the incidence of pertussis in adolescents and adults ${ }^{6}$. Other countries have not seen increases in the incidence of pertussis; France, Denmark, Sweden, and some other European countries continue to report low levels of pertussis. As highlighted in this issue, In Canada, increased pertussis activity has been reported in some provinces or regions ${ }^{10,11 .}$

Has pertussis "re-emerged"? Pertussis is a cyclical disease with peaks occurring every 2 to 5 years; is this simply an expected cyclical peak? Has surveillance improved or have there been changes to diagnostic testing? Has the organism become more virulent? Has the vaccine become less effective? How does this affect the Canadian situation?

The epidemiology of pertussis around the world may be the result of multiple different factors which with varying relevance in each country. In the United States, the California outbreak in 2010 which led to 10 infant deaths was notable for its involvement of school aged children in the 7 to 10 year age group; increased time since the preschool pertussis vaccine dose was a risk factor suggesting waning of vaccine induced immunity. A sizeable number of unimmunized children may also have contributed to the outbreak ${ }^{2}$. Duration of protection was significantly shorter in children who had received all of their pertussis vaccines with an acellular pertussis vaccine. ${ }^{1,4}$ In 2012, in Washington, Wisconsin, and Minnesota, the peak incidence of pertussis was also in school-aged children. In Washington, a short duration of protection following the pre-adolescent reduced content acellular pertussis vaccine (Tdap) was also a factor ${ }^{12}$. Thus, the US outbreaks appear to be related to shorter than expected duration of protection associated with acellular pertussis containing vaccines, receipt of all pertussis vaccines as acellular vaccine, and pockets of completely unimmunized children. In Australia, an increase in cases was reported in preschool-aged children which was attributed to the elimination of the booster dose of DTaP in the second year of life ${ }^{5,9}$. High rates of pertussis have also been reported in adults in Australia, perhaps related to the wide availability and utilization of a serological test for pertussis since the $1990 \mathrm{~s}^{9}$. In England, an outbreak of pertussis was associated with an increased number of infant deaths. The outbreak may be related to the lack of booster dose in adolescents ${ }^{6,13}$.

In considering the recent data that a single dose of whole-cell pertussis vaccine is associated with improved duration of protection, one assumes that the dose or doses of whole cell pertussis vaccine preceded the doses of acellular vaccine, since the use of acellular pertussis vaccines completely replaced whole cell vaccines in the US. It is difficult to imagine that there remains any direct protection from that initial dose or doses. Instead, it may be that protection is best when the initial dose of vaccine directs the immune system toward a Th1 type response ${ }^{14}$. Acellular pertussis vaccine, with its alum adjuvant, skews the immune system toward a Th2 biased response. In contrast, although also adjuvanted with alum, whole cell vaccine produces a Th1 biased response, similar to natural infection, perhaps related to residual presence of $B$. pertussis lipo-oligosaccharide (endotoxin), a potent Th1 adjuvant. Despite these immunological reasons why whole cell pertussis vaccines may be superior to acellular vaccines, there have also been increased reports of pertussis activity in countries using whole cell pertussis vaccine such as Brazil and Chile ${ }^{15}$. However, there is not one, single whole-cell pertussis vaccine; rather, there are multiple whole-cell pertussis vaccines and there is no correlation between their relative efficacy and the mouse intracerebral assay used as a regulatory requirement for their approval. Although data from the 
large acellular pertussis vaccine clinical trials in the 1990s suggested that the efficacy of a "good" whole cell vaccine is superior to that of the acellular pertussis vaccines, the whole cell pertussis vaccine used in two of the largest randomized controlled trials was far inferior to all of the acellular pertussis vaccines tested ${ }^{16}$. Additional information is needed about whether there are other jurisdictions using the same whole cell vaccines without these observed increases.

Concerns have also been raised that changes in Bordetella pertussis may also be contributing to the increased number of cases. Strains that have increased production of pertussis toxin have been isolated and strains that express an altered pertactin and even pertactin negative strains are becoming more common ${ }^{17,18}$. Some have suggested that these strains are emerging under immunological pressure from the current acellular pertussis vaccines. Despite the increasing isolation of these strains, there has been no clear demonstration that they are associated with increased clinical virulence or are the cause of the increased pertussis activity ${ }^{19}$.

Outbreaks have also been reported in a number of Canadian provinces. In Saskatchewan in 2010, the incidence of pertussis in children under 1 year of age rose dramatically and 6 deaths were reported; rates that exceeded those reported from California that same year. Under-immunization, particularly amongst some First Nations communities may have contributed to the increase in pertussis rates. In 2012, New Brunswick reported over 1400 cases of pertussis accounting for almost one third of the cases reported in Canada ${ }^{10}$, with an incidence that exceeded the rates reported in Washington, Wisconsin and Minnesota. In contrast to what had occurred in Saskatchewan, cases in New Brunswick were primarily focused in school-aged children and there were very few hospitalizations and no deaths amongst infants. Although a full analysis of the New Brunswick outbreak has yet to be reported, decreased duration of protection after the pre-school pertussis vaccine dose (but not the adolescent Tdap dose) as was reported in the US may have been a factor. In British Columbia, increased reports of pertussis were also received in the Fraser and Coastal health districts (Vancouver area); no increase was reported from the rest of the province. The cause for the localized resurgence of pertussis in BC is not clear; further evaluation of rates of vaccine refusal in the Vancouver area compared to other areas of the province might be worthwhile. In Ontario, a localized outbreak in the southwestern part of the province was clearly linked to a community that refuses childhood immunization ${ }^{11}$.

As observed elsewhere in the world, the epidemiology of pertussis in Canada appears to be a result of a combination of factors including the natural 2 to 5 year cycles, failure to vaccinate, and vaccine failure (both primary failure related to lower vaccine efficacy post-immunization and secondary as a result of waning immunity). What are the implications for Canada? Although to date there is no definitive demonstration that changes in the organism are leading to a resurgence of disease, it will be important to continue to monitor for this in Canada and worldwide. The shift away from culture diagnosis to PCR-based diagnosis means that monitoring of changes in the organism will be more difficult. In order for strain surveillance to continue, diagnostic laboratories need to cooperate to either culture some proportion of nasopharyngeal swabs submitted for pertussis diagnosis or to save residual secretions for later isolation of organisms for microbiological surveillance purposes. Continued surveillance of Canadian cohorts who received all of their doses with acellular vaccine is also important to further explore the duration of protection from acellular pertussis vaccine. More accurate data are required on vaccine coverage at both the local and provincial level to identify pockets of under immunization. Development of new vaccines that are more effective with a longer duration of protection is essential. Novel technologies such as live attenuated pertussis vaccines ${ }^{20}$, and vaccines with novel adjuvants that direct the immune system to a Th1 response ${ }^{21}$ may provide the solution, but are many years away. Until better vaccines are available, it will be important to make the optimal use of the vaccines currently available. High on-time vaccine coverage is still the best method we have of controlling pertussis in Canada.

\section{References}

1. Klein NP, Bartlett J, Rowhani-Rahbar A, et al. Waning protection after fifth dose of acellular pertussis vaccine in children. N Eng J Med 2012;367:1012-19. 
2. Misegades LK, Winter K, Harriman K, Talarico J, Messonnier NE, Clark TA, Martin SW. Association of childhood pertussis with receipt of 5 doses of pertussis vaccine by time since last vaccine dose, California, 2010. JAMA. 2012;308:2126-32.

3. Washington State Department of Health. 2012 Pertussis Summary for Washington State. 2013. http://www.doh.wa.gov/YouandYourFamily/lllnessandDisease/WhoopingCough.asp

4. Witt MA, Arias L, Katz PH, Truong ET, Witt DJ. Reduced risk of pertussis among persons ever vaccinated with whole cell pertussis vaccine compared to recipients of acellular pertussis vaccines in a large US cohort. Clin Infect Dis. 2013;56:1248-54.

5. Campbell P, McIntyre P, Quinn H, Hueston L, Gilbert GL, McVernon J. Increased population prevalence of low pertussis toxin antibody levels in young children preceding a record pertussis epidemic in Australia. PLoS One. 2012;7:e35874.

6. Amirthalingam G. Strategies to control pertussis in infants. Arch Dis Child. 2013;98:552-5.

7. California Department of Public Health. Pertussis (whooping cough). http://www.cdph.ca.gov/healthinfo/discond/pages/pertussis.aspx

8. Centers for Disease Control and Prevention. Pertussis outbreak trends. http://www.cdc.gov/pertussis/outbreaks/trends.html

9. Maclntyre P. Is Australia the pertussis capital of the world? National Pertussis Workshop. 25-26 August 2011, Sydney. http://www.ncirs.edu.au/news/archive.php

10. Smith T, Rotondo J, Desai S, Deehan H. Pertussis Surveillance in Canada: Trends to 2012, CCDR 2014, 40-3. http://www.phac-aspc.gc.ca/publicat/ccdr-rmtc/14vol40/dr-rm40-03/dr-rm40-03-per-eng.php

11. Deeks SL, Lim GH, Walton R, Fediurek J, Lam F, Walker C, Walters J, Crowcroft NS. Prolonged pertussis outbreak in Ontario originating in an under-immunized religious community. CCDR 2014, 40-3 Acosta A, DeBolt C, Tasslimi A, Lewis M, Stewart L, Misegades L, Messonier N, Clark T, Martin S, Patel M. Vaccine effectiveness and duration of protection of tetanus toxoid, reduced diphtheria toxoid and acellular pertussis among adolescents, Washington State, 2012. Presented at the $10^{\text {th }}$ International Symposium on Bordetella, 8-11 September 2013. Abstract O7.

12. Campbell H, Amirthalingam G, Andrews N, Fry NK, George RC, Harrison TG, Miller E. Accelerating control of pertussis in England and Wales. Emerg Infect Dis. 2012;18:38-47.

13. Ross PJ, Sutton CE, Higgins S, Allen AC, Walsh K, Misiak A, Lavelle EC, McLoughlin RM, Mills KH. Relative contribution of Th1 and Th17 cells in adaptive immunity to Bordetella pertussis: towards the rational design of an improved acellular pertussis vaccine. PLoS Pathog. 2013;9:e1003264.

14. Miranda C, Porte L, García P. Bordetella holmesii in nasopharyngeal samples from Chilean patients with suspected Bordetella pertussis infection. J Clin Microbiol. 2012;50:1505.

15. Cherry JD (2012) Why do pertussis vaccines fail? Pediatrics 129: 968-970.

16. King AJ, van der Lee S, Mohangoo A, van Gent M, van der Ark A, van de Waterbeemd B. Genome-wide gene expression analysis of Bordetella pertussis isolates associated with a resurgence in pertussis: elucidation of factors involved in the increased fitness of epidemic strains. PLoS One. 2013;8(6):e66150.

17. Queenan AM, Cassiday PK, Evangelista A (2013) Pertactin-negative variants of Bordetella pertussis in the United States. N Engl J Med 368: 583-584.

18. Bodilis $\mathrm{H}$, Guiso N. Virulence of pertactin-negative Bordetella pertussis isolates from infants, France. Emerg Infect Dis. 2013;19:471-4.

19. Kammoun H, Roux X, Raze D, Debrie AS, De Filette M, Ysenbaert T, Mielcarek N, Saelens X, Fiers W, Locht C. Immunogenicity of live attenuated B. pertussis BPZE1 producing the universal influenza vaccine candidate M2e. PLoS One. 2013;8:e59198. 
20. Polewicz M, Gracia A, Garlapati S, van Kessel J, Strom S, Halperin SA, Hancock RE, Potter AA, Babiuk LA, Gerdts V. Novel vaccine formulations against pertussis offer earlier onset of immunity and provide protection in the presence of maternal antibodies. Vaccine. 2013;31:3148-55. 